DOI: $10.17516 / 1997-1397-2020-13-6-718-732$

УДК $517.55 ; 517.9$

\title{
Upper Bounds for the Analytic Complexity of Puiseux Polynomial Solutions to Bivariate Hypergeometric Systems
}

\author{
Vitaly A. Krasikov* \\ Plekhanov Russian University of Economics \\ Moscow, Russian Federation
}

Received 10.06.2020, received in revised form 24.07.2020, accepted 20.09.2020

\begin{abstract}
The paper deals with the analytic complexity of solutions to bivariate holonomic hypergeometric systems of the Horn type. We obtain estimates on the analytic complexity of Puiseux polynomial solutions to the hypergeometric systems defined by zonotopes. We also propose algorithms of the analytic complexity estimation for polynomials.
\end{abstract}

Keywords: hypergeometric systems of partial differential equations, holonomic rank, polynomial solutions, zonotopes, analytic complexity, differential polynomial, hypergeometry package.

Citation: V.A.Krasikov,Upper Bounds for the Analytic Complexity of Puiseux Polynomial Solutions to Bivariate Hypergeometric Systems , J. Sib. Fed. Univ. Math. Phys., 2020, 13(6), 718-732.

DOI: $10.17516 / 1997-1397-2020-13-6-718-732$.

\section{Introduction and preliminaries}

The notion of complexity is widely used in Mathematics and Computer Science in the context of several various abstract objects. The computational complexity of algorithms, the algebraic complexity of polynomials, the Rademacher complexity in the computational learning theory or the social complexity in the social systems are the concepts of great importance in the corresponding fields of science. The present work is devoted to the particular type of complexity the analytic complexity of bivariate holomorphic functions.

The notion of analytic complexity is closely related to Hilbert's 13th problem, which was solved by A. N. Kolmogorov and V. I. Arnold in 1957 [1]. The initial formulation of Hilbert's 13 th problem asks whether any continuous function of several variables can be represented as a finite superposition of bivariate functions [17]. The problem of finding similar representations for analytic functions has given rise to the theory of the analytic complexity. The main objects under consideration in this theory are the analytic complexity classes.

Definition 1 (See [2]). Let $\mathcal{O}\left(U\left(x_{0}, y_{0}\right)\right)$ denote the set of holomorphic functions in an open neighborhood $U\left(x_{0}, y_{0}\right)$ of a point $\left(x_{0}, y_{0}\right) \in \mathbb{C}^{2}$. The class $C l_{0}$ of analytic functions of analytic complexity zero is defined to comprise the functions that depend on at most one of the variables. A function $f(x, y)$ is said to belong to the class $C l_{n}$ of functions with analytic complexity $n>0$ if there exists a point $\left(x_{0}, y_{0}\right) \in \mathbb{C}^{2}$ and a germ $\mathfrak{f}(x, y) \in \mathcal{O}\left(U\left(x_{0}, y_{0}\right)\right)$ of this function holomorphic at $\left(x_{0}, y_{0}\right)$ such that $\mathfrak{f}(x, y)=c(a(x, y)+b(x, y))$ for some germs of holomorphic functions $a, b \in$ $C l_{n-1}$ and $c \in C l_{0}$. If there is no such representation for any finite $n$, then the function $f$ is said to be of infinite analytic complexity.

*Krasikov.VA@rea.ru

(C) Siberian Federal University. All rights reserved 
Example 1. A generic element of the first complexity class $C l_{1}$ is a function of the form $f_{3}\left(f_{1}(x)+f_{2}(y)\right)$. A function in $C l_{2}$ can be represented in the form $f_{7}\left(f_{5}\left(f_{1}(x)+f_{2}(y)\right)+f_{6}\left(f_{3}(x)+f_{4}(y)\right)\right)$, where $f_{i}(\cdot)$ are univariate holomorphic functions, $i=1, \ldots, 7$.

For any class of analytic complexity $C l_{n}, n \in \mathbb{N}$ there exists a system of differential polynomials with constant coefficients $\Delta_{n}$ which annihilates a function if and only if it belongs to $C l_{n}$.

Example 2 (See [2]). For a bivariate function $f(x, y)$ consider the differential polynomial

$$
\Delta_{1}(f)=f_{x}^{\prime}\left(f_{y}^{\prime}\right)^{2} f_{x x y}^{\prime \prime \prime}-\left(f_{x}^{\prime}\right)^{2} f_{y}^{\prime} f_{x y y}^{\prime \prime \prime}+f_{x y}^{\prime \prime}\left(f_{x}^{\prime}\right)^{2} f_{y y}^{\prime \prime}-f_{x y}^{\prime \prime}\left(f_{y}^{\prime}\right)^{2} f_{x x}^{\prime \prime} .
$$

This differential polynomial vanishes if and only if its argument $f \in C l_{1}$.

The problem of defining whether a function belongs to an analytic complexity class is equivalent to computing the corresponding system of differential polynomials. Note that this is a problem of formidable computational complexity $[4,11]$ and a direct approach to its solution appears to be inappropriate.

An important question is a possible connection between the classes of finite analytic complexity and hypergeometric functions. In this paper we consider hypergeometric functions as solutions of hypergeometric systems in the sense of Horn $[8,10]$. We choose a matrix $A \in \mathbb{Z}^{m \times n}=\left(A_{i j}, i=1, \ldots, m, j=1, \ldots, n\right)$ and a vector of parameters $c=\left(c_{1}, \ldots, c_{m}\right) \in \mathbb{C}^{m}$. We denote the rows of this matrix by $\mathbf{A}_{i}, i=1, \ldots, m$.

Definition 2. The hypergeometric system (or the Horn system) Horn $(A, c)$ is the following system of partial differential equations:

$$
x_{j} P_{j}(\theta) f(x)=Q_{j}(\theta) f(x), j=1, \ldots, n,
$$

where

$$
\begin{aligned}
P_{j}(s) & =\prod_{i: A_{i j}>0} \prod_{l_{j}^{(i)}=0}^{A_{i j}-1}\left(\left\langle\boldsymbol{A}_{i}, s\right\rangle+c_{i}+l_{j}^{(i)}\right), \\
Q_{j}(s) & =\prod_{i: A_{i j}<0} \prod_{l_{j}^{(i)}=0}^{\left|A_{i j}\right|-1}\left(\left\langle\boldsymbol{A}_{i}, s\right\rangle+c_{i}+l_{j}^{(i)}\right),
\end{aligned}
$$

and $\theta=\left(\theta_{1}, \ldots, \theta_{n}\right), \theta_{j}=x_{j} \frac{\partial}{\partial x_{j}}$.

It has been conjectured in [14] that any hypergeometric function has finite analytic complexity. Hypergeometric systems of equations differ greatly from the differential criteria for the analytic complexity classes, but numerous computer experiments suggest that the conjecture is true in a lot of particular cases $[6,7]$. The case of hypergeometric systems with low holonomic rank has been considered in [9].

The set of functions of infinite analytic complexity is also a matter of interest. Until recently, all known examples of such functions were differentially transcendental functions, that is, functions that are not solutions to any nonzero differential polynomial with constant coefficients. Important examples of differentially algebraic functions of infinite analytic complexity have been presented in $[15,16]$.

A bivariate hypergeometric system can be defined by an integer convex polygon and a complex vector of parameters as explained in the next definition. 
Definition 3. Let $l_{i}$ denote the generator of the sublattice $\left\{s \in \mathbb{Z}^{n}:\left\langle\boldsymbol{A}_{i}, s\right\rangle=0\right\}$ and let $k_{i}$ be the number of elements in the set $\left\{\boldsymbol{A}_{1}, \ldots, \boldsymbol{A}_{m}\right\}$, which coincide with $\boldsymbol{A}_{i}$. Let us define the polygon $\mathcal{P}(A)$ (see [13]) as the integer convex polygon whose sides are translations of the vectors $k_{i} l_{i}$, the vectors $\boldsymbol{A}_{1}, \ldots, \boldsymbol{A}_{m}$ being the outer normals to its sides. We will say that the hypergeometric system $\operatorname{Horn}(A, c)$ is defined by the polygon $\mathcal{P}(A)$ and the vector $c \in \mathbb{C}$.

Definition 4. A polygon is called a zonotope if it can be represented as the Minkowski sum of segments.

In this article we investigate the analytic complexity of solutions to hypergeometric systems of equations (1) defined by zonotopes.

The present paper is organized as follows. In Section 2 we investigate particular cases of hypergeometric systems defined by zonotopes and analyze the analytic complexity of their solutions. We formulate and prove an estimate of the analytic complexity for Puiseux polynomial solutions to such systems in terms of the defining matrices and parameter vectors. In Section 3 we present algorithms for finding the supports of Puiseux polynomial solutions to hypergeometric systems and estimating the analytic complexity of polynomials. In Section 4 we consider examples of hypergeometric systems and estimate the analytic complexity of their solutions. Throughout the rest of the paper by «polynomial solutions to hypergeometric systems» we mean Puiseux polynomial solutions.

We use the Wolfram Mathematica package HyperGeometry for solving hypergeometric systems we investigate in this article. The package is available for free public use at https://www.researchgate.net/publication/318986894_HyperGeometry, the description of available functions is given in [12].

\section{Hypergeometric systems defined by zonotopes}

Let us consider the special case of hypergeometric systems defined by zonotopes. Numerous experiments suggest that the analytic complexity of polynomial solutions to such systems can be much lower than its estimate based on their supports (see [3, Proposition 4]).

The set of hypergeometric systems defined by zonotopes enjoys the following properties:

a) these systems are holonomic for generic values of parameters;

b) the holonomic rank of a hypergeometric system (see Theorem 2.5 in [5]) is given by

$$
\operatorname{rank}(\operatorname{Horn}(A, c))=d_{1} d_{2}-\sum_{\mathbf{A}_{i}, \mathbf{A}_{j}} \sum_{\text {lin. dependent }} \nu_{i j}
$$

where $d_{j}=\sum_{\substack{i=1 \\ A_{i j}>0}}^{m} A_{i j}, j=1,2$ and

$$
\nu_{i j}=\left\{\begin{array}{l}
\min \left(\left|A_{i 1} A_{j 2}\right|,\left|A_{j 1} A_{i 2}\right|\right), \text { if } \mathbf{A}_{i}, \mathbf{A}_{j} \text { are in opposite open quadrants of } \mathbb{Z}^{2}, \\
0, \text { otherwise. }
\end{array}\right.
$$

For the hypergeometric systems defined by zonotopes there is another formula for computing their holonomic rank (see Proposition 1 in [9]), which in some cases may be more suitable;

c) for any number of rows $\left(a_{i}, b_{i}\right)$ belonging to the matrix $A$ defining such a system, $A$ contains the same number of rows $\left(-a_{i},-b_{i}\right)$. Thus the rows of $A$ can be grouped into two matrices $\hat{A},-\hat{A}$. This representation is in general not unique. 
d) for a hypergeometric system defined by a zonotope one can always choose parameter values such that any solution to the resulting system is a polynomial (see [10, Proposition 6.5]). Namely, for such a hypergeometric system $\operatorname{Horn}(A, c)$, where the matrix $A$ contains $2 k$ rows, let $\alpha=\left(\alpha_{1}, \ldots, \alpha_{k}\right)$ be the part of the parameter vector $c$, corresponding to the matrix $\hat{A}$ (see the property (c) above), $\beta=\left(\beta_{1}, \ldots, \beta_{k}\right)$ be the part of this vector corresponding to $-\hat{A}$. By Proposition 4.7 in [10] the general solution to $\operatorname{Horn}(A, c)$ is a polynomial if $-\alpha_{i}-\beta_{i} \in \mathbb{N} \backslash\{0\}$ for $i=1, \ldots, k$.

The simplest instance of a zonotope is a parallelogram. The analytic complexity estimate of the solutions to the systems defined by parallelograms is the basis for more complex cases.

Proposition 1. The analytic complexity of a solution to a hypergeometric system defined by a parallelogram cannot exceed 2 .

Proof. The solutions to the hypergeometric system $\operatorname{Horn}(A, c)$ defined by a parallelogram have been described in Proposition 4.7 in [10]. For a bivariate system $(n=2)$ this formula leads to

$$
\left(x_{1}^{-a_{11}} x_{2}^{-a_{21}}\right)^{\alpha_{1}}\left(1+x_{1}^{-a_{11}} x_{2}^{-a_{21}}\right)^{-\alpha_{1}-\beta_{1}} \cdot\left(x_{1}^{-a_{12}} x_{2}^{-a_{22}}\right)^{\alpha_{2}}\left(1+x_{1}^{-a_{12}} x_{2}^{-a_{22}}\right)^{-\alpha_{2}-\beta_{2}},
$$

where $A^{-1}=\left(\begin{array}{ll}a_{11} & a_{12} \\ a_{21} & a_{22}\end{array}\right), c=\left(\alpha_{1}, \alpha_{2}, \beta_{1}, \beta_{2}\right)$. The monomials $x_{1}^{-a_{11}} x_{2}^{-a_{21}}$ and $x_{1}^{-a_{12}} x_{2}^{-a_{22}}$ both belong to $C l_{1}$, thus for any univariate analytic functions $\phi(\cdot), \psi(\cdot)$ the product $\phi\left(x_{1}^{-a_{11}} x_{2}^{-a_{21}}\right) \cdot \psi\left(x_{1}^{-a_{12}} x_{2}^{-a_{22}}\right)$ belongs to $C l_{2}$.

The following example shows that the solutions to hypergeometric systems defined by more complex polygons can still have low analytic complexity.

Example 3. A simple zonotope. Let us consider the hypergeometric system $\operatorname{Horn}\left(A^{\prime}, c^{\prime}\right)$ defined by the matrix $A^{\prime}=\left(\begin{array}{rrrrrr}1 & -1 & 1 & -1 & 0 & 0 \\ 1 & -1 & 0 & 0 & 1 & -1\end{array}\right)^{\mathrm{T}}$ and the parameter vector $c^{\prime}=$ $(-23,22,-10,0,-9,0)$. Using the formula $(2)$ we conclude that the holonomic rank of this system is equal to 3 . The hypergeometric system $\operatorname{Horn}\left(A^{\prime}, c^{\prime}\right)$ is defined by the zonotope shown in Fig. 1.
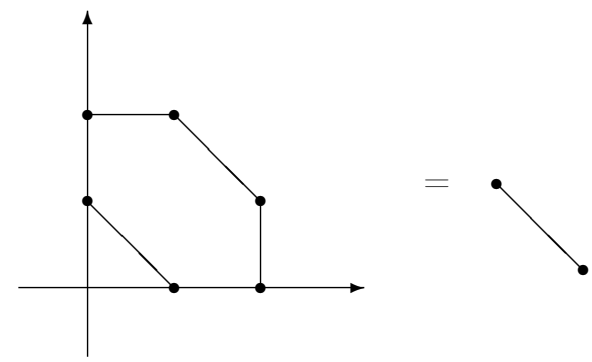

Fig. 1. Polygon defining the system $\operatorname{Horn}\left(A^{\prime}, c^{\prime}\right)$, and its representation as the Minkowski sum of segments

The support of the polynomial solutions to the system Horn $\left(A^{\prime}, c^{\prime}\right)$ is shown in Fig. 2.

Let us consider the part of the solution $p_{0}(x, y)$ whose support is bounded by the straight lines parallel to the coordinate axes. Note that $p_{0}(x, y)$ contains 110 monomials (we do not put here the whole expression due to its large size) and the known estimates for polynomials [3, Proposition 4] imply that the analytic complexity of $p_{0}(x, y)$ does not exceed 5 . Indeed, the support of $p_{0}(x, y)$ lies in the union of 10 lines parallel to the $s$ axis. The analytic complexity of the polynomial 


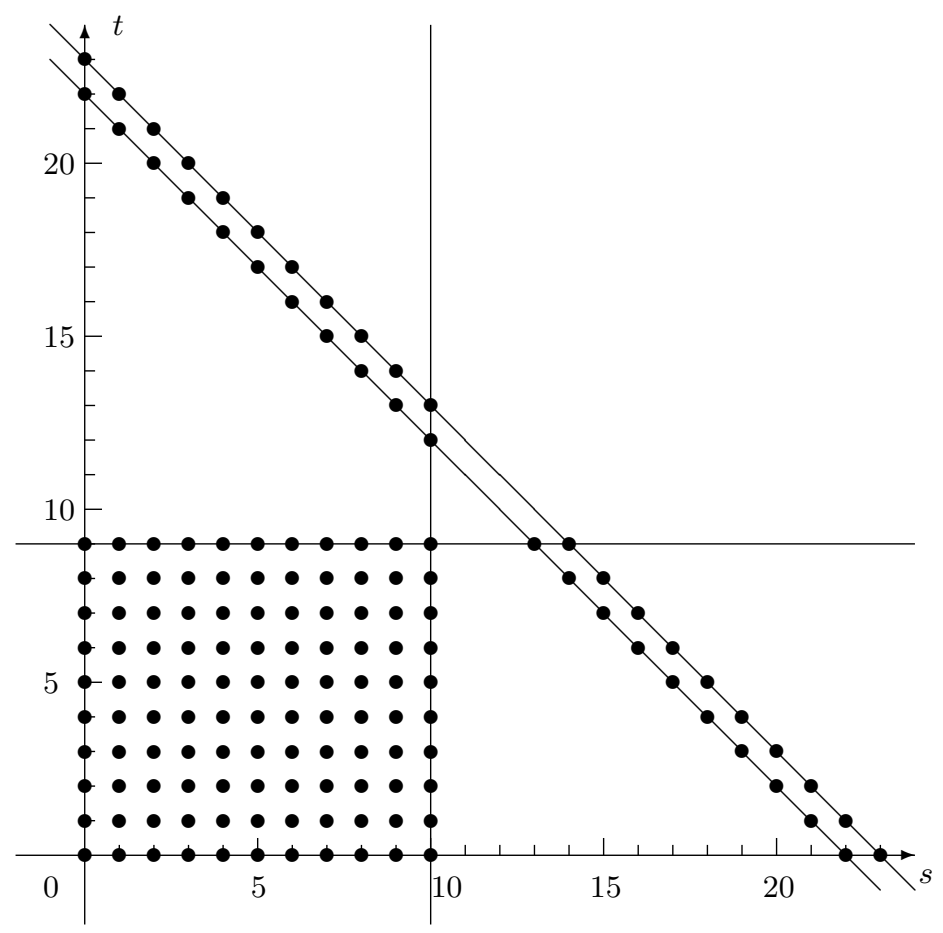

Fig. 2. The support for the solution of the system $\operatorname{Horn}\left(A^{\prime}, c^{\prime}\right)$

whose support lies on a straight line parallel to an axis cannot exceed 1 . Then the analytic complexity of the sum of $k$ such polynomials cannot exceed $1+\left\lceil\log _{2} k\right\rceil$, where by $\lceil x\rceil, x \in \mathbb{R}$ we denote the smallest integer not exceeding $x$. Later we prove that in fact the analytic complexity of $p_{0}(x, y)$ does not exceed 3 .

In general, appending a pair of rows $\left(a_{i}, b_{i}\right),\left(-a_{i},-b_{i}\right)$ to the matrix defining a hypergeometric system is equivalent to adding a pair of parallel straight lines bounding the support of the solution in the exponent space. Let the hypergeometric system be defined by a parallelogram, and let $p_{0}(x, y)=\sum_{(s, t) \in S} c_{s, t} \cdot x^{s} y^{t}$ be a polynomial solution of this system with the support $S$. Adding a pair of straight lines in the exponent space leads to the system whose solution is given by

$$
\begin{aligned}
p_{1}(x, y) & =\sum_{(s, t) \in S} \frac{\Gamma\left(\alpha_{1} s+\beta_{1} t+\gamma_{1}+1\right)}{\Gamma\left(\alpha_{1} s+\beta_{1} t+\gamma_{1}\right)} \cdot c_{s, t} \cdot x^{s} y^{t}=\sum_{(s, t) \in S}\left(\alpha_{1} s+\beta_{1} t+\gamma_{1}\right) x^{s} y^{t}= \\
& =\left(\alpha_{1} \theta_{x}+\beta_{1} \theta_{y}+\gamma_{1}\right) \sum_{(s, t) \in S} c_{s, t} x^{s} y^{t}=\left(\alpha_{1} \theta_{x}+\beta_{1} \theta_{y}+\gamma_{1}\right) p_{0}(x, y) .
\end{aligned}
$$

Using this formula repetitively we obtain the solution for $k$ additional pairs of rows $\left(a_{i}, b_{i}\right),\left(-a_{i},-b_{i}\right)$ :

$$
p_{k}(x, y)=\left(\prod_{j=1}^{k}\left(\alpha_{j} \theta_{x}+\beta_{j} \theta_{y}+\gamma_{j}\right)\right) p_{0}(x, y) .
$$

Thus the estimate for the analytic complexity of $p_{k}(x, y)$ depends on the analytic complexity of $p_{0}(x, y)$. This dependence is described in detail in the following Proposition and its corollaries. Recall that we use the notation $\theta_{x}=x \frac{\partial}{\partial x}, \theta_{y}=y \frac{\partial}{\partial y}$ and $\alpha_{j}, \beta_{j}, \gamma_{j} \in \mathbb{C}, j=1, \ldots, k$. 
Proposition 2. If $f(x, y) \in C l_{n}$ then $\left(\alpha \theta_{x}+\beta \theta_{y}+\gamma\right) f(x, y) \in C l_{2 n+1}$.

Proof. We use induction by $n$ to show that $\left(\alpha \theta_{x}+\beta \theta_{y}\right) f(x, y) \in C l_{2 n}$.

For $n=1$ we can represent $f(x, y)$ in the form $f(x, y)=c(a(x)+b(y))$.

$$
\left(\alpha \theta_{x}+\beta \theta_{y}\right) c(a(x)+b(y))=c^{\prime}(a(x)+b(y)) \cdot\left(\alpha x a^{\prime}(x)+\beta y b^{\prime}(y)\right),
$$

and this function belongs to $\mathrm{Cl}_{2}$ as a product of $C l_{1}$ functions. If the statement holds for all $n<N$, and $f(x, y)$ belongs to $C l_{N}$, which means it can be represented as $f(x, y)=h\left(f_{1}(x, y)+\right.$ $\left.f_{2}(x, y)\right)$, where $f_{1}(x, y), f_{2}(x, y) \in C l_{N-1}$, then

$$
\begin{gathered}
\left(\alpha \theta_{x}+\beta \theta_{y}\right) h\left(f_{1}(x, y)+f_{2}(x, y)\right)= \\
=h^{\prime}\left(f_{1}(x, y)+f_{2}(x, y)\right)\left(\left(\alpha \theta_{x}+\beta \theta_{y}\right) f_{1}(x, y)+\left(\alpha \theta_{x}+\beta \theta_{y}\right) f_{2}(x, y)\right) .
\end{gathered}
$$

Both of the functions $f_{1}(x, y)$ and $f_{2}(x, y)$ belong to $C l_{N-1}$, so the estimate of the analytic complexity for $\left(\alpha \theta_{x}+\beta \theta_{y}\right) f_{i}(x, y), i=1,2$ is $C l_{2 N-2}$. Then their sum belongs to $C l_{2 N-1}$ and, after the multiplication of the result by $h^{\prime}\left(f_{1}(x, y)+f_{2}(x, y)\right) \in C l_{N}$, the product belongs to $C l_{2 N}$. Thus we conclude that for any $n$, if $f(x, y) \in C l_{n}$ then $\left(\alpha \theta_{x}+\beta \theta_{y}\right) f(x, y) \in C l_{2 n}$. Adding $\gamma f(x, y) \in C l_{n}$ to this expression we obtain a function in $C l_{2 n+1}$.

Corollary 1. For any $f(x, y) \in C l_{n}$ the analytic complexity of

$$
\left(\prod_{j=1}^{k}\left(\alpha_{j} \theta_{x}+\beta_{j} \theta_{y}+\gamma_{j}\right)\right) f(x, y)
$$

cannot exceed $2^{k}(n+1)-1$.

Corollary 2. Assume that the analytic complexity of a polynomial solution $p_{0}(x, y)$ to the hypergeometric system $\operatorname{Horn}(\tilde{A}, \tilde{c})$ does not exceed $n, S$ is a support of $p_{0}(x, y)$. Let the matrix $A$ be obtained from $\tilde{A}$ by appending $k$ pairs of vectors $\left(a_{i}, b_{i}\right),\left(-a_{i},-b_{i}\right)$, vector c be obtained from $\tilde{c}$ by appending $2 k$ elements. Then the analytic complexity of a polynomial solution with the support $S$ to the hypergeometric system $\operatorname{Horn}(A, c)$ does not exceed $2^{k}(n+1)-1$.

Example 3. (Continued). Let us use Corollary 2 to estimate the analytic complexity of a solution to the system Horn $\left(A^{\prime}, c^{\prime}\right)$. To do this, consider the system $\operatorname{Horn}\left(\tilde{A}^{\prime}, \tilde{c}^{\prime}\right)$, defined by the matrix $\tilde{A}^{\prime}=\left(\begin{array}{rrrr}1 & 0 & 0 & -1 \\ 0 & 1 & -1 & 0\end{array}\right)^{\mathrm{T}}$ and the vector of parameters $\tilde{c}^{\prime}=(-10,-9,1,1)$. This system differs from the original one only by the absence of the pair of straight lines with the normal vectors $(1,1)$ and $(-1,-1)$ bounding the support of the solution. Thus this support for the system $\operatorname{Horn}\left(\tilde{A}^{\prime}, \tilde{c}^{\prime}\right)$ coincides with the support of $p_{0}(x, y)$. Note that this system is defined by a parallelogram and hence by Proposition 2 the analytic complexity of its solutions cannot exceed 2. Computations show that the basis in the space of solutions to the system $\operatorname{Horn}\left(\tilde{A}^{\prime}, \tilde{c}^{\prime}\right)$ consists of the single function $(x-1)^{10}(y-1)^{9} \in C l_{1}$, and hence $p_{0}(x, y) \in C l_{3}$ by Corollary 2. The supports of two other solutions to Horn $\left(A^{\prime}, c^{\prime}\right)$ lie on two parallel straight lines, so a linear combination of these solutions belongs to $\mathrm{Cl}_{3}$, and the general solution to Horn $\left(A^{\prime}, c^{\prime}\right)$ is a function in $\mathrm{Cl}_{4}$.

The following theorem is the main theoretical result of the paper. It contains the general estimate of the analytic complexity for polynomial solutions to hypergeometric systems defined by zonotopes. 
Theorem 1. Let $\operatorname{Horn}(A, c)$ be a hypergeometric system defined by a zonotope. Assuming that the matrix $A$ contains $2 k$ rows, consider the matrices $\hat{A}$ and $-\hat{A}$ such that the union of their rows coincides with the set of rows of $A$. Let $\alpha$ be a part of the parameter vector $c$ corresponding to the matrix $\hat{A}, \beta$ be a part of this vector corresponding to $-\hat{A}$, and define the vector $\hat{c}=\left(\hat{c}_{1}, \ldots, \hat{c}_{k}\right)$ by $\hat{c}_{i}=-\alpha_{i}-\beta_{i}$.

If $\hat{c}_{i} \in \mathbb{N} \backslash\{0\}, i=1, \ldots, k$, then the analytic complexity of the general solution to $\operatorname{Horn}(A, c)$ does not exceed

$$
\min \left(3 \cdot 2^{k-2}-1+\left\lceil\log _{2} \frac{k(k-1)}{2}\right\rceil, 2+\left\lceil\log _{2}\left(\max _{i=1, \ldots, k} \hat{c}_{i}+1\right)\right\rceil+\left\lceil\log _{2}(k-1)\right\rceil\right) .
$$

Proof. For any system defined by a parallelogram the condition $\hat{c}_{i} \in \mathbb{N} \backslash\{0\}$ provides the existence of a polynomial solution (see [10, Proposition 4.7] and the proof of Proposition 2.). Appending of the rows $\left(a_{i}, b_{i}\right),\left(-a_{i},-b_{i}\right)$ to the matrix defining the hypergeometric system affects only the coefficients of this solution but not its support. Without loss of generality we can choose a vector of parameters $c$ such that the support of the general solution to $\operatorname{Horn}(A, c)$ coincides with a union of supports of the solutions to a finite number of systems defined by parallelograms (see proof of Proposition 6.5 in [10]). Thus the condition $\hat{c}_{i} \in \mathbb{N} \backslash\{0\}$ provides the existence of a polynomial basis in the space of solutions to $\operatorname{Horn}(A, c)$.

The matrix $A$ contains $2 k$ rows, so supports of the solutions are bounded by $k$ pairs of straight lines. Let us assign a natural number from 1 to $k$ to each pair of lines. The union of these supports is a subset of $\frac{k(k-1)}{2}$ parallelogram intersections (it is the sum of an arithmetic progression), each intersection we denote as $\square_{i, j}$, where $i \in\{1, \ldots, k\}$ and $j \in\{1, \ldots, k\}$ are numbers assigned to pairs of straight lines which form the intersection, $i<j$. For any $(i, j) \in\{1, \ldots, k\}^{2}$ the solution to $\operatorname{Horn}(A, c)$ whose support lies in the intersection $\square_{i, j}$ belongs to $C l_{3 \cdot 2^{k-2}-1}$ (by Corollary 2). The analytic complexity of the sum of $\frac{k(k-1)}{2}$ functions in $C l_{3 \cdot 2^{k-2}-1}$ (that is, the analytic complexity of the general solution to $\operatorname{Horn}(A, c))$ cannot exceed $\operatorname{estim}_{1}\left(\bigcup_{i, j=1}^{k} \square_{i, j}\right)=$ $=3 \cdot 2^{k-2}-1+\left\lceil\log _{2} \frac{k(k-1)}{2}\right\rceil$ (see $[3$, Section 5$\left.]\right)$.

On the other hand, there is the estimate based on the number of parallel straight lines containing the points of the support (see Proposition 4 in [3]). While the analytic complexity of any polynomial with the support belonging to a straight line does not exceed 2 , the number of these lines corresponding to the $i$-th row of $\hat{A}$ equals $\hat{c}_{i}+1$. Thus for any $i$ the analytic complexity of the part of the solution whose support belongs to $\bigcup_{j=1}^{k} \square_{i, j}$ cannot exceed $2+\left\lceil\log _{2}\left(\max _{i=1, \ldots, k} \hat{c}_{i}+1\right)\right\rceil$. Note that there is no need to use all of $k$ pairs of bounding straight lines to estimate the analytic complexity of the general solution this way, since $k-1$ pairs already bound the whole support of the solution. The sum of $k-1$ elements in $C l_{2+\left\lceil\log _{2}\left(\max _{i=1, \ldots, k} \hat{c}_{i}+1\right)\right\rceil}$ cannot exceed $\operatorname{estim}_{2}\left(\bigcup_{i, j=1}^{k} \square_{i, j}\right)=2+\left\lceil\log _{2}\left(\max _{i=1, \ldots, k} \hat{c}_{i}+1\right)\right\rceil+\left\lceil\log _{2}(k-1)\right\rceil$. The minimal of the numbers $\operatorname{estim}_{1}\left(\bigcup_{i, j=1}^{k} \square_{i, j}\right)$, estim ${ }_{2}\left(\bigcup_{i, j=1}^{k} \square_{i, j}\right)$ is the sought estimate.

An example of using the estimate given in Theorem 1 is shown in Fig. 3. Note that there are three sets of parallel lines, each corresponding to one of the $\hat{c}_{i}$. For each of the parallelogram intersections there are 2 estimates: $\operatorname{estim}_{1}\left(\square_{i, j}\right)$, based on Corollary 2 and estim ${ }_{2}\left(\square_{i, j}\right)$ based on Proposition 4 in [3]. 


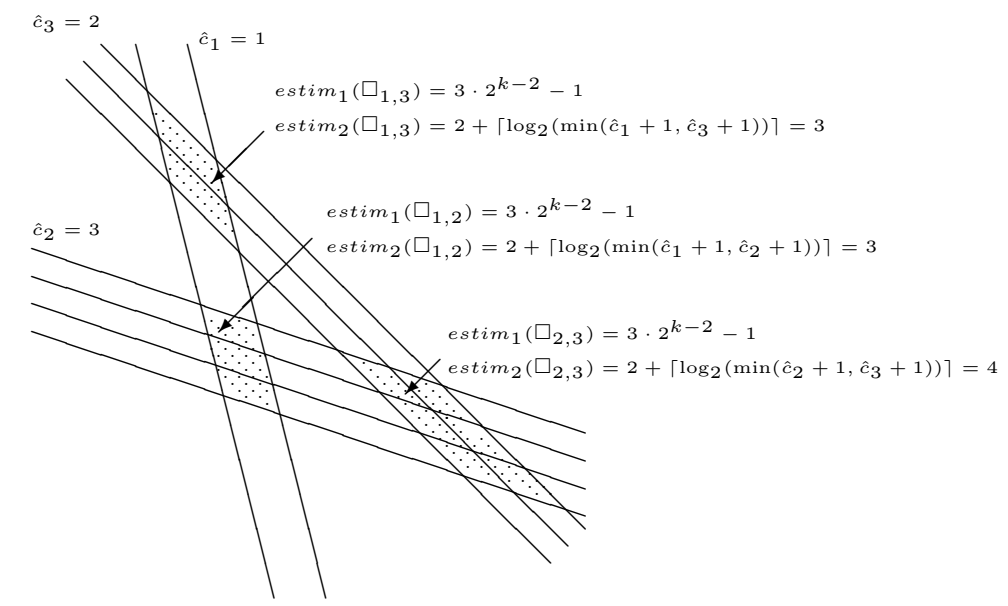

Fig. 3. The analytic complexity estimate for a polynomial solution to a simple hypergeometric system

We order $\hat{c}_{i}$ by the ascension and choose $v$ to be a vector with the elements $v_{i}=$ $=\min \left(2+\left\lceil\log _{2}\left(\hat{c}_{i}+1\right)\right\rceil, 3 \cdot 2^{k-2}-1+\left\lceil\log _{2}(k-i)\right\rceil\right), i=1, \ldots, k-1$. To find more accurate value for the analytic complexity estimate from Theorem 1, one could use Algorithm 2 from Section 3 using $v$ as an input vector. The general estimate from Theorem 1 can be rough, if values of $\hat{c}_{i}$ vary greatly for different $i$. For example in Fig. 3 estim ${ }_{2}\left(\square_{1,2}\right)=$ estim $_{2}\left(\square_{1,3}\right)=3$, $\operatorname{estim}_{2}\left(\square_{2,3}\right)=4$, and for the general estimate we use the maximal of these values. The vector $v$ in this case provides the choice of the better estimate.

\section{Algorithms of analytic complexity estimation}

The following algorithm allows one to compute the analytic complexity of any given bivariate polynomial.

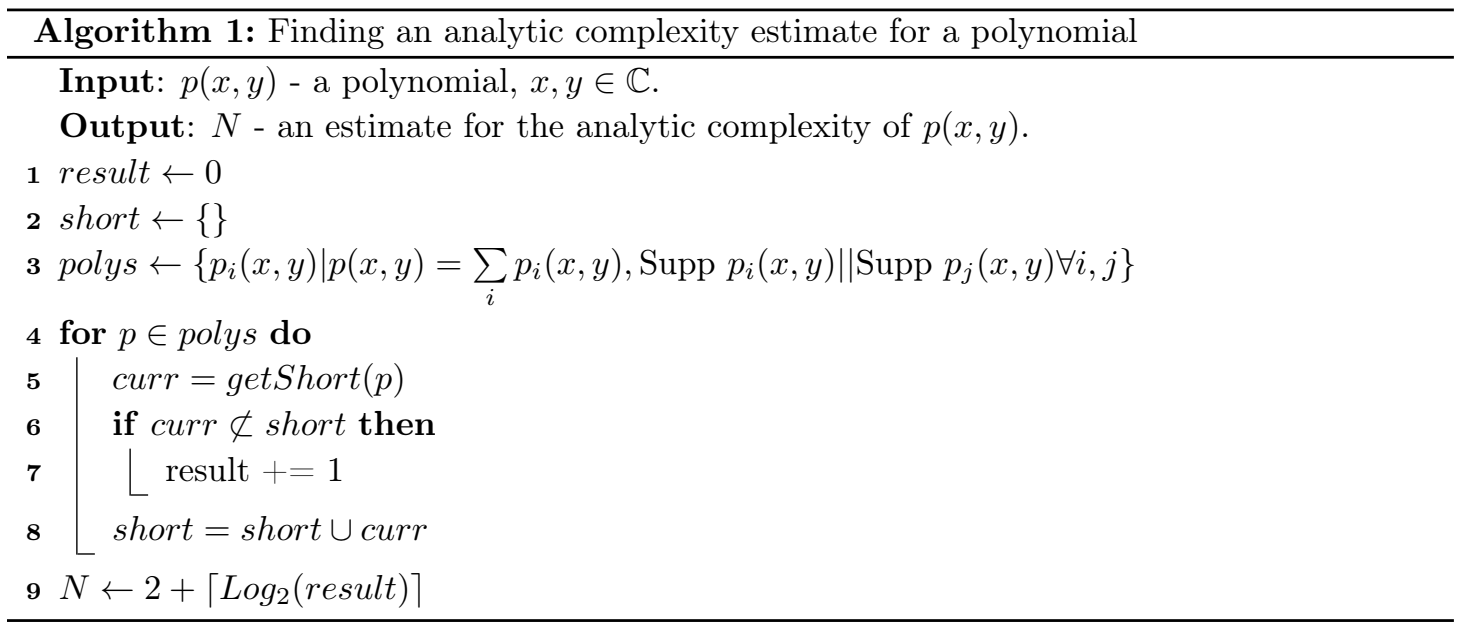

The main advantage of this algorithm compared to the existing ones is its ability to distinct the powers of lower degree polynomials included in the original polynomial as summands. Without this feature, even the analytic complexity of the function like $p(a(x)+b(y)) \in C l_{1}$, where $p(t), a(x), b(y)$ are univariate polynomials, is estimated based on its support, which becomes very 
inaccurate with the growth of degree of $p(t)$.

The input of the function getShort() is a homogeneous polynomial and the output contains elements of its decomposition into the sum of powers. Note that the definition of polys assumes the ambiguity of the representation of the polynomial as the sum of finitely many polynomials supported in parallel straight lines. Any of such representations yields an estimate, but some of them may be better than the other ones.

To estimate the analytic complexity of the general solution to the hypergeometric system from Theorem 1 one can use the following algorithm.

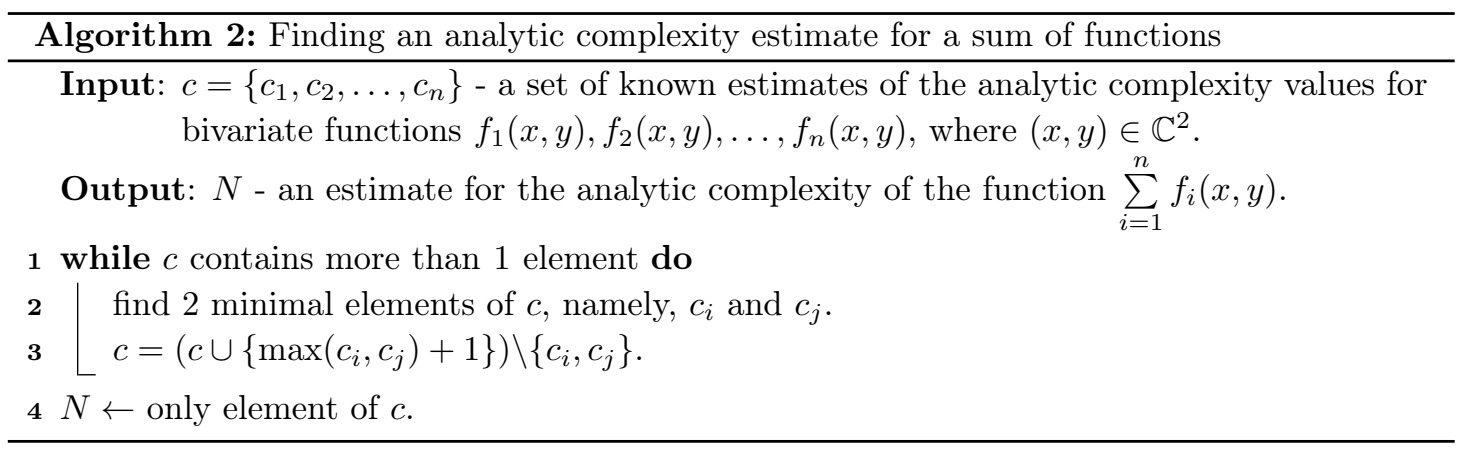

Algorithm 2 is finite, since at each step the number of elements in $c$ decreases by 1 .

The following algorithm allows one to find the support of a polynomial solution to a given hypergeometric system defined by a zonotope, provided that such a solution exists. The algorithm is based on Proposition 4.7 in [10].

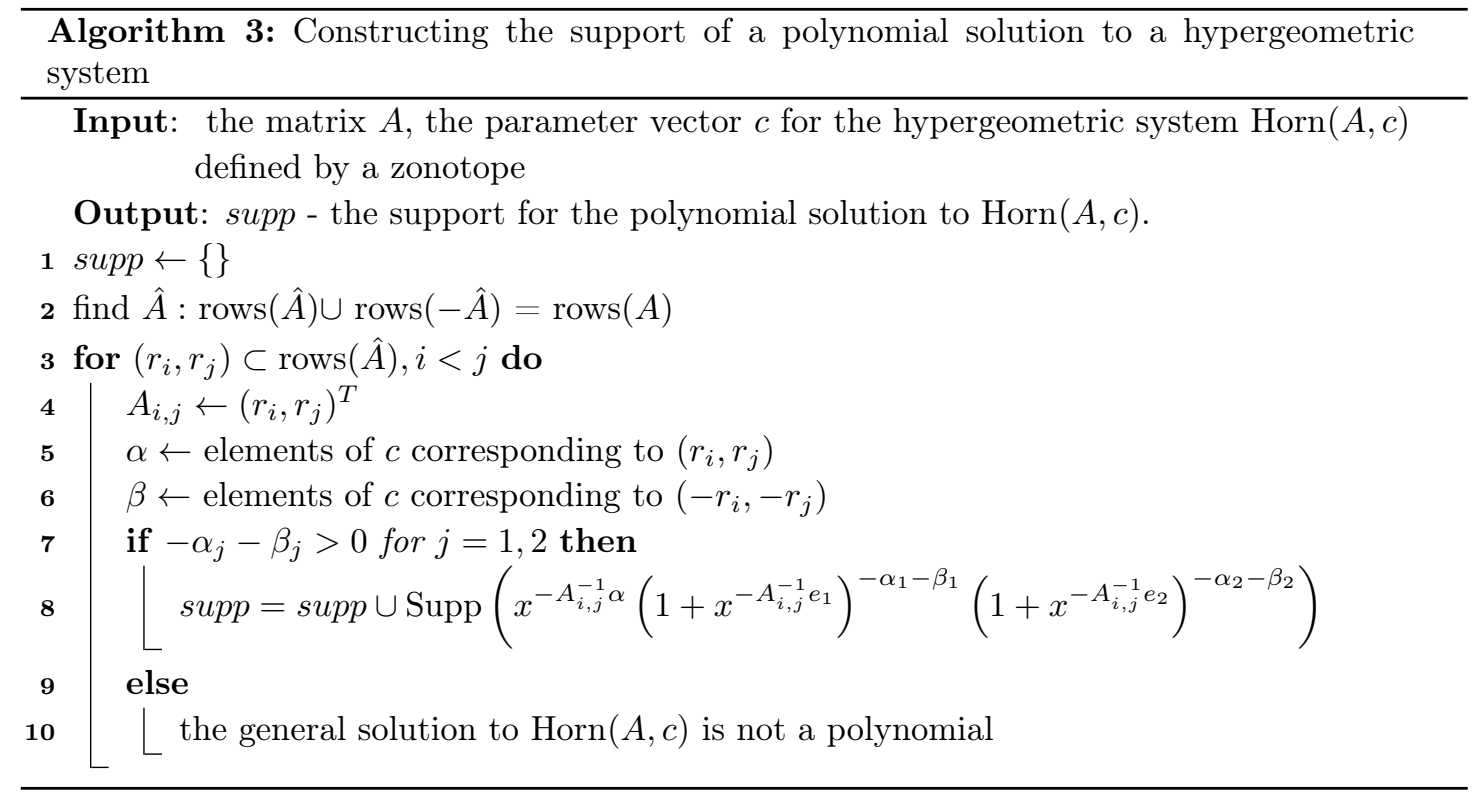

For some pairs of rows $r_{i}, r_{j}$ the solution to the corresponding system defined by a parallelogram is not a polynomial. In this case, a part of the basis in the solution space can still consist of polynomials, and their supports can be found by means of Algorithm 3 .

\section{Examples}

Example 3 (Continued). Let us replace the parameter vector $c^{\prime}$ in the system $\operatorname{Horn}\left(A^{\prime}, c^{\prime}\right)$ by 
the vector $(k, 0,0,0,0,0)$. The corresponding system is given by

$$
\begin{aligned}
& x \theta_{x}\left(\theta_{x}+\theta_{y}+k\right)-\theta_{x}\left(\theta_{x}+\theta_{y}\right), \\
& y \theta_{y}\left(\theta_{x}+\theta_{y}+k\right)-\theta_{y}\left(\theta_{x}+\theta_{y}\right) .
\end{aligned}
$$

A basis in its solution space is given by $1, \log \frac{x}{x-1}+\sum_{j=1}^{k-1} \frac{(-1)^{j}}{j(x-1)^{j}}, \log \frac{y}{y-1}+\sum_{j=1}^{k-1} \frac{(-1)^{j}}{j(y-1)^{j}}$, so there is no polynomial basis for these parameter values. Nevertheless, the analytic complexity of the general solution is equal to 1 .

The present example shows that the analytic complexity of solutions to hypergeometric systems can be heavily dependent on parameter vectors defining these systems. A resonant choice of their parameters can drastically reduce the analytic complexity of general solutions to such systems.

Example 4. An octagon zonotope. Consider Example 6.8 in [10]. In order to find the analytic complexity of a polynomial solution to the hypergeometric system defined by the matrix

$$
A=\left(\begin{array}{rrrrrrrr}
1 & -1 & -1 & 1 & -3 & 3 & 2 & -2 \\
2 & -2 & 1 & -1 & -2 & 2 & -1 & 1
\end{array}\right)^{\mathrm{T}}
$$

and the vector of parameters $c=(3,-5,-2,1,-2,-1,-1,-1)$ we can use the basis of the solutions to this system, computed in [10]. There are 3 solutions whose analytic complexity is equal to 2 , and 28 solutions in $C l_{1}$, two of them also belonging to $C l_{0}$. Therefore the analytic complexity of the general solution to this system cannot exceed 7 . Note that this estimate is based on a trivial grouping of the basis functions into pairs, but the very specific structure of the solution support makes it possible to show that the analytic complexity does not exceed 6 .

Let us estimate the analytic complexity of the general solution to this system using Theorem 1 . The vector $\hat{c}$, ordered by the ascension, is $(1,2,2,3)$. Then the vector $v=(3,4,4)$ (it includes only support-based estimates, because of low values of the elements of $\hat{c}$ ), and, by using Algorithm 2, we conclude that the general solution belongs to $\mathrm{Cl}_{6}$.

Futhermore, we can estimate the analytic complexity of a solution to any hypergeometric system we obtain by appending a pair of rows to $A$ (the only condition is that these rows are not collinear to the rows of $A$ ). Note that this estimate does not depend much on the difference between new parameters. If this difference is big, it becomes the last element of the ordered vector $\hat{c}$, and does not affect the new vector $v$, the new element of the vector $v$ is equal to $2+\left\lceil\log _{2}(3+1)\right\rceil=4$, and the resulting analytic complexity is 6 . On the contrary, if this difference is low, for example, if it is equal to 1 , the new vector $\hat{c}=(1,1,2,2,3)$, the new vector $v=(3,3,4,4)$, and the analytic complexity is also equal to 6 . Thus we conclude that appending 2 rows to the matrix $A$ does not affect the analytic complexity of the solution to the system.

Example 5. A decagon zonotope. Consider the hypergeometric system $\operatorname{Horn}\left(A_{1}, c_{1}\right)$, defined by the matrix

$$
\left(\begin{array}{rrrrrrrrrr}
-1 & 1 & 0 & 0 & -2 & 2 & 3 & -3 & 3 & -3 \\
0 & 0 & -1 & 1 & 1 & -1 & 1 & -1 & 2 & -2
\end{array}\right)^{\mathrm{T}}
$$

and the parameter vector $c_{1}=(-1,0,4,-5,1,-4,-9,6,-4,0)$. The zonotope defining the matrix 3 is shown in Fig. 4.

By Theorem 2.5 in [5] the holonomic rank of the system Horn $\left(A_{1}, c_{1}\right)$ equals 34 . The support to the solution to this system computed by the means of Algorithm 3 is shown in Fig. 5.

A polynomial basis in the solution space to $\operatorname{Horn}\left(A_{1}, c_{1}\right)$ consists of the 4 monomials $\frac{x^{6}}{y^{9}}, \frac{x^{17 / 3}}{y^{8}}, \frac{x^{3}}{y^{3}}, \frac{x^{8 / 3}}{y^{2}}$ and 30 polynomials 


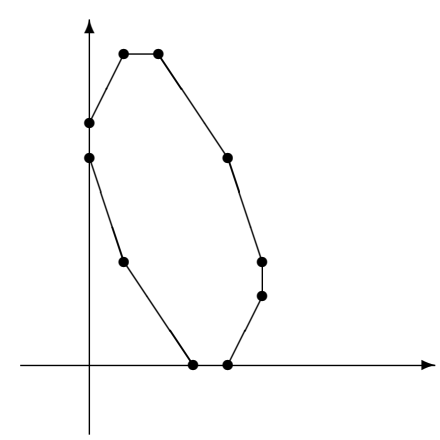

Fig. 4. The zonotope which defines the matrix (3)

$$
\begin{aligned}
& \frac{1}{x y^{6}}+\frac{5643}{637 x y^{5}}+\frac{247095}{8281 x y^{4}}+\frac{329460}{8281 x y^{3}}+\frac{27455}{286 y^{4}}+\frac{82365}{49 y^{3}}+\frac{741285}{49 y^{2}}+\frac{724812}{7 y} \\
& \frac{20 y^{3}}{63 x}-\frac{4 y^{2}}{35 x}+\frac{4 y^{2}}{5}-\frac{18 y}{5}+1, \quad \frac{3 y^{3 / 2}}{380 x}-\frac{3969 y^{5 / 2}}{41990 x}+\frac{1323 y^{7 / 2}}{16796 x}-\frac{51}{55} y^{3 / 2}+\sqrt{y}, \\
& -\frac{11 y^{12}}{115311 x}-\frac{33 y^{11}}{38437 x}-\frac{297 y^{10}}{100555 x}-\frac{24 y^{9}}{5915 x}+\frac{3 y^{9}}{1105}+\frac{y^{8}}{26}+\frac{36 y^{7}}{143}+y^{6}, \quad x y^{4}-\frac{2}{13} x y^{5}, \\
& \frac{8 y^{5}}{99 x}+\frac{4 y^{4}}{3 x}+\frac{50 y^{5}}{81}+y^{4}, \quad \frac{1550775 x^{7 / 2} y^{5}}{82808479}-\frac{31465 x^{9 / 2} y^{5}}{61400001}+x^{5 / 2} y^{4}-\frac{5175 x^{7 / 2} y^{4}}{89947}, \\
& \frac{1547 x^{4} y^{5}}{103455}-\frac{91 x^{4} y^{4}}{6840}-\frac{91 x^{3} y^{5}}{1026}+x^{3} y^{4}, \quad \frac{806 y^{5}}{129 x^{8 / 3}}+\frac{84656 y^{4}}{735 x^{5 / 3}}+\frac{y^{4}}{x^{8 / 3}}, \quad \frac{x^{13 / 3}}{y^{6}}+\frac{451 x^{13 / 3}}{261 y^{5}}, \\
& \frac{87 y^{5}}{82 x^{7 / 3}}+\frac{5220 y^{5}}{275561 x^{10 / 3}}+\frac{36575 y^{4}}{2392 x^{4 / 3}}+\frac{y^{4}}{x^{7 / 3}}, \quad \frac{44 y^{5}}{1183 x^{3}}+\frac{33 y^{5}}{182 x^{2}}+\frac{y^{4}}{x^{2}}, \quad \frac{x^{16 / 3}}{y^{8}}+\frac{1378 x^{16 / 3}}{451 y^{7}}, \\
& -\frac{21}{46} x^{2 / 3} y^{5}+x^{2 / 3} y^{4}+\frac{119}{286} x^{5 / 3} y^{4}, \quad-\frac{12}{247} x^{4 / 3} y^{5}+x^{4 / 3} y^{4}-\frac{364 \sqrt[3]{x} y^{5}}{1045}, \quad \frac{2 x}{7 y}+x, \\
& \frac{11985}{299} x^{8 / 7} y^{2 / 7}+\frac{14382 x^{8 / 7}}{253 y^{5 / 7}}+\frac{x^{8 / 7}}{y^{12 / 7}}, \quad \frac{1200 x^{2 / 7}}{1643 y^{3 / 7}}+\frac{345 x^{9 / 7}}{31 y^{3 / 7}}+\frac{x^{9 / 7}}{y^{10 / 7}}, \quad \frac{x^{10 / 3}}{y^{4}}+\frac{261 x^{10 / 3}}{238 y^{3}}, \\
& \frac{114774 x^{6 / 7} y^{5 / 7}}{28405}+\frac{1188 x^{6 / 7}}{65 y^{2 / 7}}+\frac{x^{6 / 7}}{y^{9 / 7}}, \quad \frac{x^{10 / 7}}{y^{8 / 7}}+\frac{731 x^{3 / 7}}{638 \sqrt[7]{y}}+\frac{1763 x^{10 / 7}}{754 \sqrt[7]{y}}, \\
& \frac{x^{4 / 7}}{y^{6 / 7}}+\frac{32680 x^{11 / 7}}{8613 y^{6 / 7}}+\frac{1558}{261} x^{4 / 7} \sqrt[7]{y}, \quad \frac{169}{150} x^{5 / 7} y^{3 / 7}+\frac{x^{5 / 7}}{y^{4 / 7}}+\frac{65 x^{12 / 7}}{136 y^{4 / 7}}, \\
& -\frac{1}{66} 5 x^{2} y^{3}+\frac{5}{7} x^{2} y^{2}-\frac{45}{28} x^{2} y+x^{2}, \quad x^{11 / 5} y^{2 / 5}-\frac{4301 x^{11 / 5} y^{7 / 5}}{4277}+\frac{232254 x^{11 / 5} y^{12 / 5}}{1056419}, \\
& x^{9 / 5} y^{3 / 5}-\frac{1287 x^{9 / 5} y^{8 / 5}}{1634}+\frac{55913 x^{9 / 5} y^{13 / 5}}{346408}, \quad x^{12 / 5} y^{4 / 5}-\frac{68}{19} x^{7 / 5} y^{9 / 5}-\frac{116}{231} x^{12 / 5} y^{9 / 5}, \\
& x^{8 / 5} y^{6 / 5}+\frac{5824 x^{13 / 5} y^{6 / 5}}{432837}-\frac{1064 x^{8 / 5} y^{11 / 5}}{2829}, \quad \frac{x^{5}}{y^{7}}+\frac{8 x^{5}}{15 y^{6}}-\frac{21 x^{4}}{55 y^{6}}-\frac{182 x^{4}}{15 y^{5}}-\frac{91 x^{4}}{24 y^{4}}, \\
& \frac{x^{14 / 3}}{y^{7}}+\frac{828 x^{14 / 3}}{85 y^{6}}-\frac{585488 x^{11 / 3}}{48825 y^{5}}+\frac{21758 x^{14 / 3}}{23715 y^{5}}-\frac{2488324 x^{11 / 3}}{35805 y^{4}} .
\end{aligned}
$$

There are 14 functions in $C l_{1}$ and 20 functions in $C l_{2} \backslash C l_{1}$ among these polynomials.

The analytic complexity estimate of the general solution to $\operatorname{Horn}\left(A_{1}, c_{1}\right)$ obtained by grouping these functions into pairs is $C l_{7}$. Theorem 1 gives a better estimate: since $\hat{c}=(2,2,3,3,4)$, 


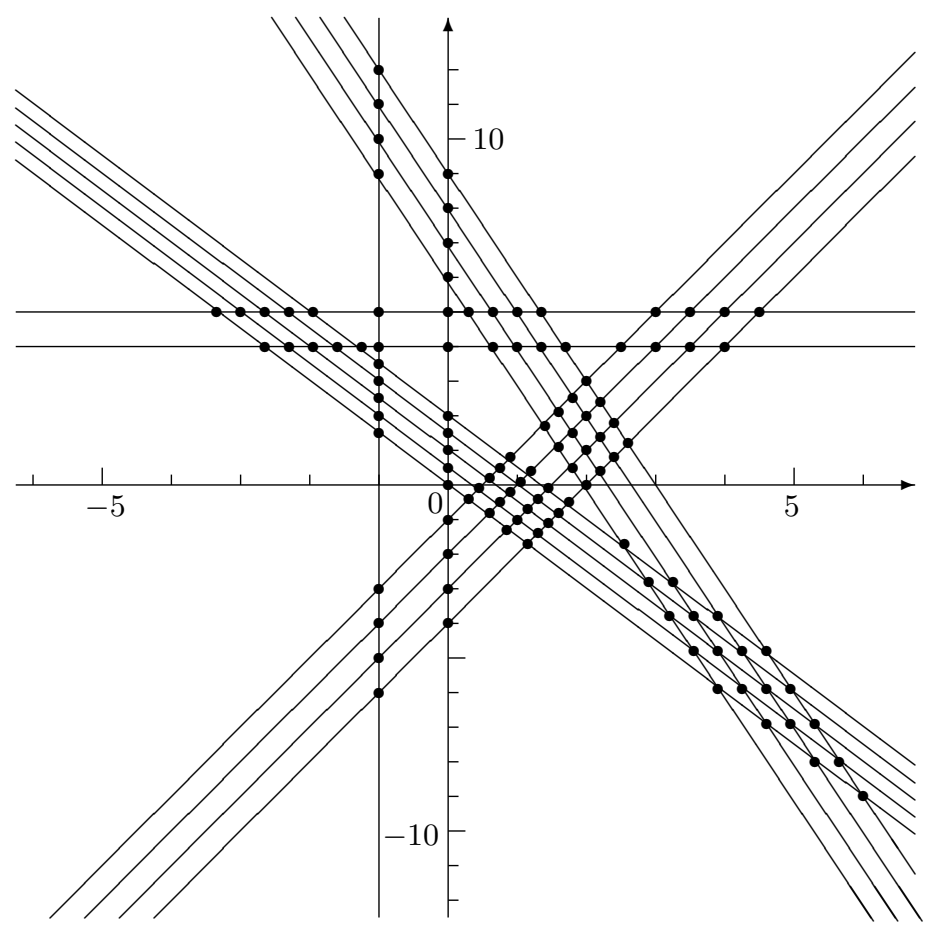

Fig. 5. The support for the solution of the system $\operatorname{Horn}\left(A_{1}, c_{1}\right)$

$v=(4,4,4,4)$, it follows that the general solution belongs to $C l_{6}$.

The following examples present hypergeometric systems defined by polygons other than zonotopes whose solutions have low analytic complexity.

Example 6. A pentagon. The matrix $\left(\begin{array}{rrrrrrr}1 & -1 & 0 & 1 & -1 & 0 & 0 \\ 1 & 0 & -1 & 0 & 0 & -1 & 1\end{array}\right)^{\mathrm{T}}$ and the vector of parameters $(-4,0,0,-1,-2,-1,-2)$ define the hypergeometric system

$$
\begin{aligned}
& x\left(\theta_{x}+\theta_{y}-4\right)\left(\theta_{x}-1\right)-\theta_{x}\left(\theta_{x}-2\right), \\
& y\left(\theta_{x}+\theta_{y}-4\right)\left(\theta_{y}-1\right)-\theta_{y}\left(\theta_{y}-2\right) .
\end{aligned}
$$

This system is holonomic and its holonomic rank equals 4 . The pure basis (see [10]) in its solution space is given by the Taylor polynomials

$x^{2} y^{2}, \quad 1-4 x-4 y+12 x y, \quad 6 x^{2}-4 x^{3}+x^{4}-12 x^{2} y+4 x^{3} y, \quad 6 y^{2}-12 x y^{2}-4 y^{3}+4 x y^{3}+y^{4}$.

The first and the second of these polynomials belong to $C l_{1}$, the third and the fourth belong to $\mathrm{Cl}_{2}$. Thus the general solution is a function in $\mathrm{Cl}_{4}$.

Example 7. A trapezoid, high holonomic rank. A basis in the solution space of the hypergeometric system with holonomic rank $k$ defined by the operators

$$
\begin{aligned}
& x \theta_{x}^{k-1}\left(\theta_{x}+\theta_{y}\right)-(-1)^{k} \theta_{x}^{k}, \\
& y\left(\theta_{x}+\theta_{y}\right)+\theta_{y} .
\end{aligned}
$$

is given by $\left\{\log ^{j}((y+1) / x), j=0, \ldots, k-1\right\}$ (see Fig. 6). The generating solution equals $\log ^{k-1}((y+1) / x)$. Thus the general solution to this system belongs to $C l_{1}$ by the conservation principle. This example shows that the analytic complexity of solutions to hypergeometric systems with high holonomic rank can still be low. 


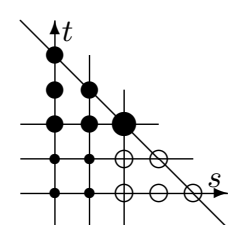

a)

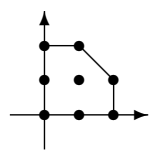

b)

Fig. 6. a) the supports of solutions to the system (4); b) polygon defining the system (4)

Example 8. A triangle with no symmetries. The hypergeometric system

$$
\begin{aligned}
& x\left(\theta_{x}+\theta_{y}-4\right)\left(\theta_{x}+2 \theta_{y}-4\right)-\left(2 \theta_{x}+3 \theta_{y}-4\right)\left(2 \theta_{x}+3 \theta_{y}-5\right), \\
& y\left(\theta_{x}+\theta_{y}-4\right)\left(\theta_{x}+2 \theta_{y}-4\right)\left(\theta_{x}+2 \theta_{y}-3\right)-\left(2 \theta_{x}+3 \theta_{y}-4\right)\left(2 \theta_{x}+3 \theta_{y}-5\right)\left(2 \theta_{x}+3 \theta_{y}-6\right)
\end{aligned}
$$

is holonomic and its holonomic rank equals 6 . The pure basis in its solution space is given by the Laurent polynomials

$$
\begin{gathered}
x^{-4} y^{4}, \quad x^{-2} y^{3}, \quad x^{7} y^{-3}, \quad x^{8} y^{-4}, \quad 3 y^{2}+2 x^{-1} y^{2} \\
6 x^{2}+12 x^{3}+x^{4}+4 x^{5} y^{-2}+6 x^{6} y^{-2}-12 x^{4} y^{-1}-4 x^{5} y^{-1}-12 x y-4 x^{2} y .
\end{gathered}
$$

In the Fig. 7 the small filled circles correspond to monomial solutions, the two empty circles indicate the binomial solution and the big filled circles correspond to the remaining polynomial solution. The analytic complexity of the general solution to the system (7) does not exceed 5 .

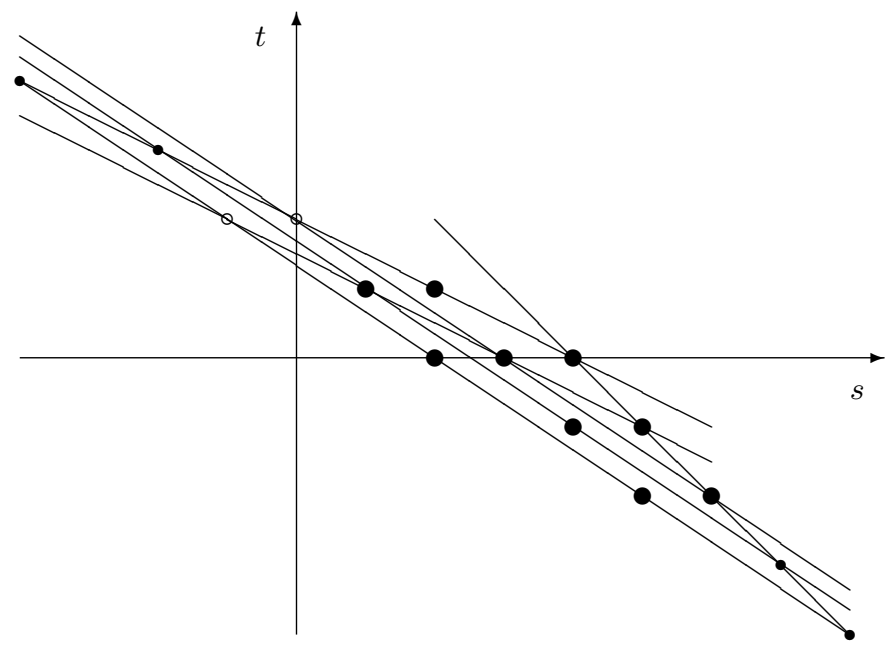

Fig. 7. The supports of solutions to the system (5)

This research was performed in the framework of the state task in the field of scientific activity of the Ministry of Science and Higher Education of the Russian Federation, project "Development of the methodology and a software platform for the construction of digital twins, intellectual analysis and forecast of complex economic systems", Grant no. FSSW-2020-0008. 


\section{References}

[1] V.I.Arnold, On the representation of continuous functions of three variables by superpositions of continuous functions of two variables, Sbornik Mathematics, 48(1959), no. 1, 3-74.

[2] V.K.Beloshapka, Analytic complexity of functions of two variables, Russian J. Math. Phys., 14(2007), no. 3, 243-249.

[3] V.K.Beloshapka, Analytical complexity: Development of the topic, Russian J. Math. Phys., 19(2012), no.4, 428-439.

[4] V.K.Beloshapka, On the complexity of differential algebraic definition for classes of analytic complexity, Math. Notes, 105(2019), no. 3, 323-331.

[5] A.Dickenstein, L.F.Matusevich, T.M.Sadykov, Bivariate hypergeometric D-Modules, $A d-$ vances in Mathematics, 196(2005), 78-123.

[6] A.Dickenstein, T.M.Sadykov, Algebraicity of solutions to the Mellin system and its monodromy, Dokl. Math., 75(2007), no. 1, 80-82. DOI: 10.1134/S106456240701022X

[7] A.Dickenstein, T.M.Sadykov, Bases in the solution space of the Mellin system, Sbornik Mathematics, 198(2007), no. 9, 1277-1298.

[8] J.Horn, Über die Konvergenz der hypergeometrischen Reihen zweier und dreier Veränderlichen, Math. Ann., 34(1889), 544-600.

[9] V.A.Krasikov, Analytic complexity of hypergeometric functions satisfying systems with holonomic rank two, Lecture Notes in Computer Science, 11661(2019), 330-342.

[10] T.M.Sadykov, S.Tanabe, Maximally reducible monodromy of bivariate hypergeometric systems, Izv. Math., 80(2016), no. 1, 221-262.

[11] T.M.Sadykov, Beyond the first class of analytic complexity, Lecture Notes in Computer Science, $11077(2018), 335-344$.

[12] T.M.Sadykov, Computational problems of multivariate hypergeometric theory, Programming and Computer Software, 44(2018), no. 2, 131-137. DOI: 10.1134/S0361768818020093

[13] T.M.Sadykov, The Hadamard product of hypergeometric series, Bulletin des Sciences Mathematiques, 126(2002), no. 1, 31 .

[14] T.M.Sadykov, On the analytic complexity of hypergeometric functions, Proceedings of the Steklov Institute of Mathematics, 298(2017), no. 1, 248-255.

DOI: 10.1134/S0081543817060165

[15] M.A.Stepanova, Analytic complexity of differential algebraic functions, Sbornik Mathematics, 210(2019), no. 12, 1774-1787.

[16] M.A.Stepanova, On analytical complexity of antiderivatives, Journal of Siberian Federal University. Mathematics \& Physics, 12(2019), no. 6, 694-698.

DOI: 10.17516/1997-1397-2019-12-6-694-698

[17] A.G.Vitushkin, On Hilbert's thirteenth problem and related questions, Russian Math. Surveys, 59(2004), no. 1, 11-25. 


\title{
Верхние границы аналитической сложности решений двумерных гипергеометрических систем в классе многочленов Пюизо
}

Виталий А. Красиков

Российский экономический университет им. Г. В. Плеханова Москва, Российская Федерация

\begin{abstract}
Аннотация. В статье исследуется аналитическая сложность решений двумерных голономных гипергеометрических систем типа Горна. Получены оценки аналитической сложности решений в классе многочленов Пюизо для гипергеометрических систем, заданных зонотопами. Также предложены алгоритмы для оценки аналитической сложности многочленов.

Ключевые слова: гипергеометрические системы дифференциальных уравнений в частных производных, голономный ранг, полиномиальные решения, зонотопы, аналитическая сложность, дифференциальный многочлен.
\end{abstract}

